\title{
1 Compact Grating-Coupled Biosensor for the Analysis of Thrombin
}

\author{
2 Daria Kotlarek ${ }^{\dagger}$, Mariia Vorobii, ${ }^{\ddagger}$ Wojciech Ogieglo, ${ }^{\ddagger}{ }^{\S}$ Wolfgang Knoll ${ }^{\dagger}$, Cesar Rodriguez-Emmeneg- \\ 3 ger, ${ }^{\dagger}$ Jakub Dostálek ${ }^{\dagger} *$. \\ ${ }^{\dagger}$ Biosensor Technologies, AIT-Austrian Institute of Technology GmbH, Konrad-Lorenz-Straße 24, 3430 Tulln an der \\ Donau, Austria \\ $\$$ DWI - Leibniz Institute for Interactive Materials and Institute of Technical and Macromolecular Chemistry, RWTH Aachen University, \\ Forckenbeckstraße 50, 52074 Aachen, Germany \\ $\S$ Current address: Advanced Membranes \& Porous Materials Center, 4700 King Abdullah University of Science and Technology \\ (KAUST), Thuwal 23955-6900, Kingdom of Saudi Arabia
}

10 Supporting Information Placeholder

11 KEYWORDS: surface plasmon resonance, grating-coupled surface plasmon resonance, biosensor, thrombin, antifouling 12 brushes, aptamer, blood plasma, point of care

14

15
ABSTRACT: A compact optical biosensor for direct detection of 48 thrombin in human blood plasma (HBP) is reported. This biosensor 49 platform is based on wavelength spectroscopy of surface plasmons 50 on a chip with a periodically corrugated gold film that carries an 51 antifouling thin polymer layer consisting of poly[( $N$-(2-hydroxy- 52 propyl) methacrylamide)-co-(carboxybetaine methacrylamide) ${ }_{53}^{53}$ poly(HPMA-co-CBMAA) brushes. This surface architecture pro- 54 vides superior resistance to nonspecific and irreversible adsorption 55 of abundant compounds in the analyzed HBP samples in compari- 56 son to standard surface modifications. The carboxylate groups 57 along the polymer brushes were exploited for the covalent immo- 58 bilization of aptamer ligands. These ligands were selected to spe- 59 cifically capture target thrombin analyte from the analyzed HBP 60 sample in a way that does not activate the coagulatory process at 61 the biosensor surface with poly(HPMA-co-CBMAA) brushes. Di- 62 rect label-free analysis of thrombin in the medically relevant con- 63 centration range $(1-20 \mathrm{nM})$ is demonstrated without the need for 64 diluting the HBP samples or using additional steps for signal en- 65 hancement. The reported platform constitutes the first step toward 66 a portable and sensitive point-of-care (POC) device for direct de- 67 tection of thrombin in human blood.

Hemostasis is an indispensable physiological mechanism that 72 maintains the integrity of the vascular system and circulation of the 73 blood in the fluid state. ${ }^{1}$ It relies on a number of highly regulated 74 pro- and anti-thrombotic pathways that control the suppression of 75 bleeding at the site of vessel injury, clot dissolution, and wound 76 remodeling. ${ }^{2}$ The delicate balance between coagulation and fibri- 77 nolytic activity can be disrupted by congenital and acquired coag- 78 ulopathies, ${ }^{1}$ anticoagulation therapy, ${ }^{3}$ surgical procedures, ${ }^{4,5}$ cardi- 79 opulmonary bypass, ${ }^{6}$ and extracorporeal life support (ECLS). ${ }^{7}$ In 80 these cases, the patient is exposed to a risk of life-threatening hem- 81 orrhage or a thrombotic event that requires immediate medical in- 82 tervention. The incidence of hemostatic complications and their 83 consequences (mortality, number of transfusions, time spent in the 84 intensive care, cost of the treatment) can be substantially reduced by routine clinical diagnostics complemented by self-testing of a patient's coagulation status. ${ }^{8-10}$ Yet, because of the complexity of the hemostatic system with a plentiful positive and negative feedback controls, a generic and widely accepted method for the assessment of coagulation stage does not exist neither at patient's home nor in the clinical practice. ${ }^{11}$

Affinity optical biosensors based on surface plasmon resonance (SPR) are considered as one of the prime candidates for the "next generation' diagnostic devices. ${ }^{12}$ The SPR biosensors hold potential to serve in emerging POC applications offering the advantage of fast response, real-time measurement, and already developed implementations to portable devices. ${ }^{13}$ Up to now, portable systems based on SPR biosensor principle were pursued for the applications in food safety, ${ }^{14,15}$ environmental monitoring, biodefense, ${ }^{16,17}$ and medical diagnostics. ${ }^{18,19}$ The majority of the reported portable SPR devices rely on the Kretschmann configuration of the attenuated total reflection (ATR) enabling resonant excitation of propagating surface plasmons (PSPs) on sensor surface with continuous metallic film. ${ }^{20,21}$ In order to simplify the use of SPR sensor chips in compact or portable reader devices, diffraction grating-coupled surface plasmon resonance (GC-SPR) provides an alternative to ATR method. ${ }^{22,23}$ The GC-SPR chips carry nanostructures which can be fabricated by scaled up means by injection molding, ${ }^{24}$ rollto-roll nanoimprint lithography, ${ }^{25}$ or laser interference lithography. ${ }^{26}$ These types of nanostructures were integrated to disposable sensor chips and used in the compact sensor designs supported by dedicated readers ${ }^{27-29}$ as well as by optics integrated to smartphones. ${ }^{30}$

Throughout the last years, we witnessed a gradual progress in the development of thrombin biosensors based on electrochemical, ${ }^{31}$ piezoelectric ${ }^{32}$ and optical transducers. ${ }^{33}$ Among these, SPR biosensors reached the limit of detection at pico- and attomolar concentrations ${ }^{34,35}$ and relied on readers with miniature design by using plasmonic nanostructures ${ }^{36}$ or optical fibers. ${ }^{37}$ Although detection of thrombin in medically relevant range $(5-20 \mathrm{nM})$ is well established in model samples, the operation in complex biological media 
such as human blood, plasma, and serum remains challenging due 64 to the unspecific adsorption of matrix proteins to the sensor surface 65 - a phenomenon also called 'fouling'. The fouling from blood 66 plasma is the result of complex cooperative intermolecular interac- 67 tions, ${ }^{38}$ which constitutes a multiscale process that begins with ad- 68 sorption of smaller proteins that are subsequently replaced by more surface-affine ones (Vroman effect ${ }^{39}$ ) and followed by the activa- 69 tion of different coagulatory factors as well as thrombocytes. In the SPR affinity biosensors, fouling severely complicates the discrim- 70 ination between the response originating from the capture of the 71 target analyte and the unspecific protein adsorption on the sensor 72 surface, and thus, impairs their performance. The most common 73 approach to reduce the protein fouling is a modification of the me- 74 tallic surface with self-assembled monolayers (SAM) carrying ol- 75 igo(ethylene glycol) moieties..$^{40}$ This modification typically pro- 76 vides good resistance to the fouling from model samples, however, 77 it is not sufficient when the sensor surface is challenged with more 78 complex bodily fluids. ${ }^{41}$ Often used strategy to mitigate the effect 79 of unspecific interactions is diluting the analyzed sample ten to 80 hundreds of times with a reaction buffer, ${ }^{42,43}$ which lowers the con- 81 centration of the target analyte that is available for the detection by 82 the same factor. In addition, there were reported multiple-step as- 83 says with the enhancement of specific response by using sandwich 84 assay format combined with metallic nanoparticles ${ }^{43}$ and advanced 85 reference-compensated measurement strategies were pursued. ${ }^{44} 86$ Despite these advances in SPR biosensor technology, only one of 87 the aforementioned studies achieved sufficient limit of detection 88 (LOD) of thrombin in $10 \%$ serum for the prediction of the throm- 89 botic event by the combined aptamer-based rolling circle amplifi- 90 cation and bio-bar-coded AuNP enhancement. ${ }^{35}$

Notably, the SPR analysis of complex biological media can be 93 simplified by the implementation of anti-fouling biointerface archi- 94 tecture that will resist the biological matrix effect and thus enable 95 the specific detection of the target analyte without the need of sam- 96 ple dilution or signal amplification. The poly[( $N$-(2-hydroxypro- 97 pyl) methacrylamide)-co-(carboxybetaine methacrylamide) 97 poly(HPMA-co-CBMAA) brushes have become one of the most 98 successful strategies for eliminating protein adsorption on the sen -99 sor surface from blood serum and plasma ${ }^{45-47}$ and saliva. ${ }^{48}$ Mos $\$ 00$ importantly, the immobilization of the receptor molecules to thes 01 poly(HPMA-co-CBMAA) brushes induces only minute changes in 02 their structure and thus it minimally impairs their anti-fouling prop 103 erties. This functionality was demonstrated for brushes prepared by 104 statistical copolymerization of two anti-fouling monomers HPMA 105 and CBMAA $(85: 15 \%)$ that provides a small fraction of side group 06 available for functionalization with receptors..$^{45}$

In this study, we address the important clinical need for a com 108 pact device to monitor the concentration of thrombin in biologica 10 media by developing a compact plasmonic biosensor. It should bq 11 emphasized that the detection of thrombin in the blood plasma ig 12 particularly challenging because its capture on the sensor surface 13 locally increases its concentration and can trigger the coagulation 14 cascade. Therefore, the surface of a diffraction grating was coated 15 with an advanced biointerface architecture comprising 16 poly(HPMA-co-CBMAA) brushes that were post modified with 17 aptamer ligand. The single-stranded DNA aptamer used in thi 17 study binds to exosite I of thrombin by adopting a G-quadruple 18 conformation leading to inhibition of thrombin-catalyzed clot for 119 mation. ${ }^{49,50}$ In addition, we used an univalent thrombin inhibitor 120 argatroban in order to prevent the coagulation of the bulk humar 21 blood plasma (HBP) sample. The argatroban binds to the active site 22 of thrombin blocking the catalytic activity of soluble and fibrin 123 bound thrombin and reducing thrombin-mediated activation of platelets. ${ }^{51}$ By occupying the active site only, the argatroban does not impair the recognition of thrombin by the surface-attached aptamer. The importance of an anti-fouling sensor surface engineering is demonstrated by selective capture of medically relevant concentrations of thrombin in undiluted HBP.

\section{EXPERIMENTAL}

Materials. Thrombin purified from human plasma $\left(\mathrm{M}_{\mathrm{w}}=37 \mathrm{kDa}\right)$ was purchased from Enzo Life Sciences (Switzerland). The thrombin binding aptamer HD1 (amino modifier C6 5'TTT TTG GTT GGT GTG GTT GG-3') and control aptamer (scrambled sequence: amino modifier C6 5'- TTT TTG GTG GTG GTT GTG GT-3') were synthesized by Integrated DNA Technologies (Belgium). The pooled normal HBP samples were obtained from Innovative Research (USA). Alkane PEG-thiol with a carboxylic acid group (carboxyl PEG-thiol, TH 003-m11.n6-0.1) was purchased from Prochimia (Poland) and (11-mercaptoundecyl) triethyleneglycol (hydroxyl PEG-thiol, SPT-0011) was purchased from SensoPath Technologies Inc. (USA). Buffer solutions were prepared by using ultrapure water (arium pro, Sartorius Stedim) with all reagents used as received unless otherwise stated. 2-(NMorpholino) ethanesulfonic acid (MES), acetic acid, sodium acetate (SA), sodium chloride, HEPES, PBS buffer tablets and argatroban monohydrate $\left(\mathrm{Mw}_{\mathrm{w}}=526.65 \mathrm{~g} / \mathrm{mol}\right)$ were purchased from Sigma-Aldrich (Austria). 1-Ethyl-3-(3-dimethylaminopropyl)-carbodiimide (EDC) and $N$-hydroxysuccinimide (NHS) were obtained from Thermo Scientific (Austria). $\mathrm{CuCl}_{2}$ (99.999\%), 1,4,8,11-tetramethyl-1,4,8,11-tetraazacyclotetradecane (Me4) Cyclam, 98\%) were purchased from Sigma-Aldrich (Germany). $\mathrm{CuCl}$ was purchased from abcr $\mathrm{GmbH}$ (Germany). Methanol (MeOH), ethanol (EtOH) were purchased from VWR Chemicals (Germany). Milli$\mathrm{Q}$ water for the polymer synthesis was obtained using Elga ${ }^{\mathrm{TM}} \mathrm{US}$ filter Purelab Plus UF purification system (PL5113 02) (United Kingdom)

Preparation of plasmonic gratings. A master structure of crossed relief grating was prepared by UV laser interference lithography as described before. ${ }^{52}$ It was cast to polydimethylsiloxane (PDMS, Sylgard 184 from Dow Corning, USA), which was cured overnight at $60{ }^{\circ} \mathrm{C}$. The PDMS structure was detached from the master and used as a working stamp for further replication by UV nanoimprint lithography. The UV-curable polymer Amonil MS10 (Amo GmbH, Germany) was spun on a BK7 glass substrate at $3000 \mathrm{rpm}$ for $120 \mathrm{~s}$. The PDMS working stamp was placed on the top of the Amonil layer and the polymer was irradiated with UV light at $\lambda=365 \mathrm{~nm}$ with the irradiation dose of $2 \mathrm{~J} / \mathrm{cm}^{2}$ (UV lamp Bio-Link 365, Vilber Lourmat). Then, the PDMS stamp was released from the UV-cured Amonil layer leaving an imprinted pattern of the master structure on the BK7 glass substrate. Subsequently, chromium (4 $\mathrm{nm}$ thickness) and gold (100 nm thickness) layers were deposited on the Amonil grating by vacuum thermal evaporation (HHV AUTO 306 from HHV LTD, UK) in vacuum better than $10^{-6} \mathrm{mbar}$. The prepared GC-SPR sensor chips comprised an area that was structured and area that was flat and served as a reference in the optical measurements.

Synthesis of poly(HPMA-co-CBMAA) brushes. $\Omega$-mercaptoundecyl bromoisobutyrate and $N$-(2-hydroxypropyl) methacrylamide (HPMA) were synthesized according to the literature ${ }^{53,54}$ (3-methacryloylaminopropyl) - (2-carboxyethyl) -dimethylammonium (carboxybetaine methacrylamide, CBMAA) was synthesized using a modification of procedure reported earlier. ${ }^{55}$ Shortly, the GC-SPR sensor chips with the gold film were immersed in $2.4 \mathrm{mM}$ 
1 solution of $\omega$-mercaptoundecyl bromoisobutyrate in EtOH over2 night to form SAM of the initiator. For the atom transfer radical polymerization (ATRP), $8 \mathrm{~mL}$ of $\mathrm{MeOH}$ and $8 \mathrm{~mL}$ of Milli-Q water were degassed in separated Schlenk flasks via seven freeze-
5 pump-thaw cycles. Simultaneously, catalyst $\left[\mathrm{CuCl}_{2}(6.1 \mathrm{mg}\right.$, $678 \mu \mathrm{mol}), \mathrm{CuCl}(20.5 \mathrm{mg}, 207 \mu \mathrm{mol})$, and $\mathrm{Me}_{4} \mathrm{Cyclam}(70.6 \mathrm{mg}$, $7275 \mu \mathrm{mol})]$ and monomers

a)
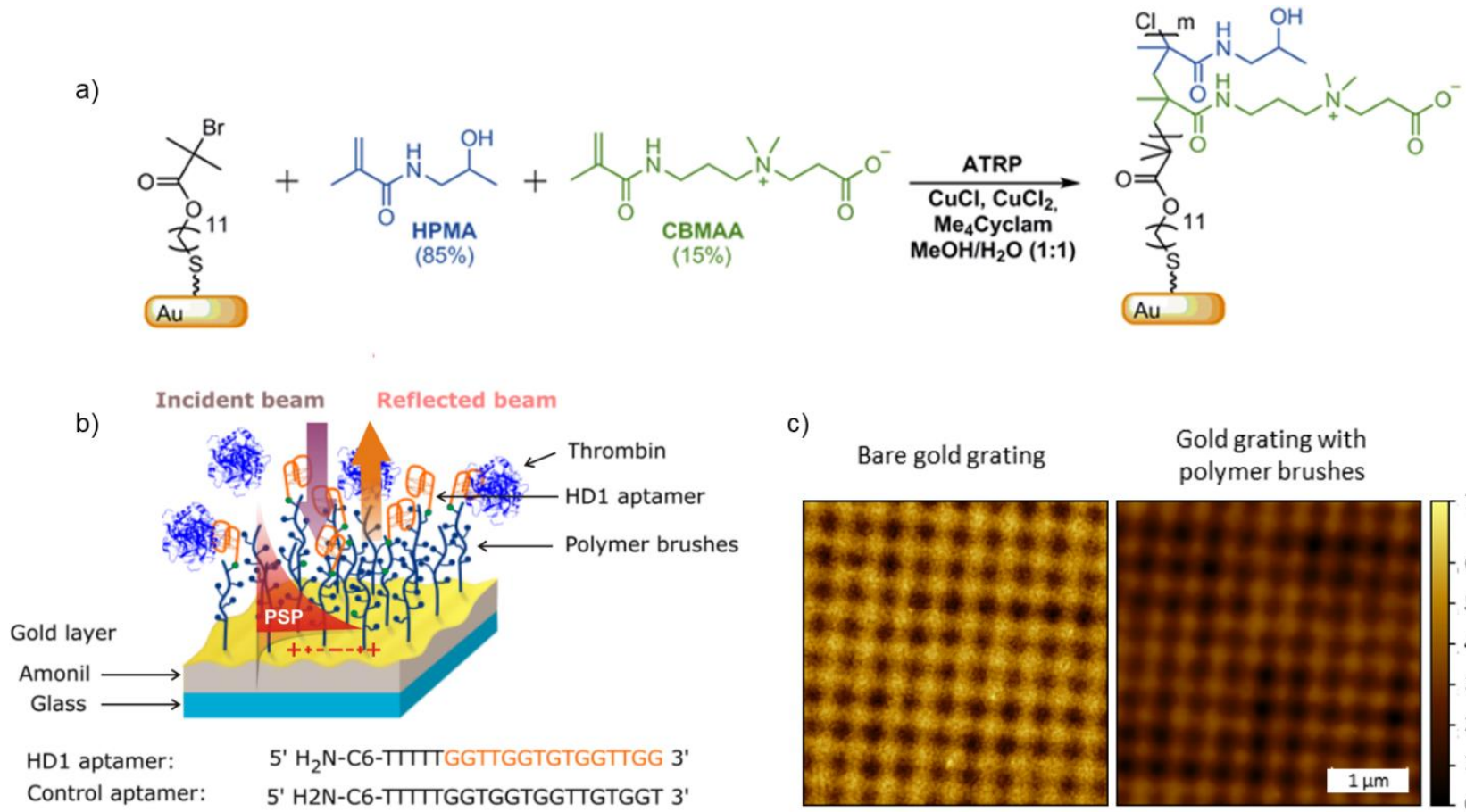

Gold grating with polymer brushes

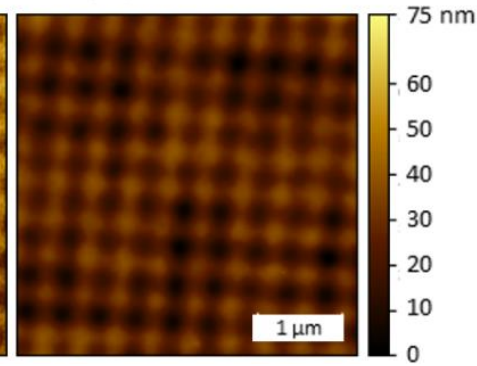

Figure 1. a) Synthesis and chemical structure of poly(HPMA-co-CBMAA) brushes on a gold surface by ATRP. b) Sensor chip surface with corrugated gold layer carrying nonfouling poly(HPMA-co-CBMAA) brushes for detection of thrombin. c) The AFM observation of the bare gold grating and the grating coated with polymer brushes.

[HPMA (1.4 g, $9.7 \mathrm{mmol})$ and CBMAA (409 mg, $1.7 \mathrm{mmol})$ ] 28 were placed in separated Schlenk flasks and degassed by a pump 29 and refilled. Subsequently, $4 \mathrm{~mL}$ of $\mathrm{MeOH}$ and $7 \mathrm{~mL}$ of Milli-Q 30 water together with $3 \mathrm{~mL}$ of $\mathrm{MeOH}$ were transferred to the Schlenk 31 flasks containing catalyst and monomers, respectively, under $\mathrm{N}_{2}$ at- 32 mosphere and were stirred until all solids were dissolved. Then, the 33 obtained catalyst solution was mixed with the monomer solution 34 using a gas-tight syringe under $\mathrm{N}_{2}$ atmosphere and transferred to 35 previously degassed reactors containing GC-SPR sensor chip with 36 SAM. The polymerization was carried out at $30^{\circ} \mathrm{C}$ for $2 \mathrm{~h}$. The 37 samples were taken out from the reactors, washed with $\mathrm{EtOH}, 38$ Milli-Q water and dried with $\mathrm{N}_{2}$. The scheme of the polymerization 39 is shown in Figure. 1a.

Morphology measurements. The morphology of the crossed 41 relief grating before and after the modification with poly(HPMA- 42 co-CBMAA) brushes was observed by using atomic force micros- 43 copy (AFM, PicoPlus from Molecular Imaging, Agilent Technolo- 44 gies, Germany) in the tapping mode with tips PPP-NCHR-50 (Na- 45 nosensors, Switzerland). The acquired images were analyzed with 46 the open-source software Gwyddion (version 2.47 from 47 gwyddion.net). The grafting of polymer brushes was monitored by 48 measuring the thickness of dried polymer film using a spectro- 49 scopic ellipsometer M2000 (J.A.Woollam Co., USA) operated in 50 rotating compensator mode. All measurements were performed in 51 air at room temperature in the wavelength range of 350-1000 nm 52 with a Xe-arc lamp light source and angle of incidence of 65, 70 and $75^{\circ}$. The obtained data were analyzed with the CompleteEASE software using a transparent Cauchy-type optical dispersion to model the polymer brushes.

Optical setup of GC-SPR biosensor. The in situ probing of the sensor surface was performed by using an in-house developed compact instrument (Figure. 2). The polychromatic light from a halogen light source (12V, HL-2000, Mikropack, USA) was coupled to an input arm of Y-optical fiber splitter (Ø $400 \mu \mathrm{m}$ core, 0.39 NA, Thorlabs, UK). The light emitted from the output arm of Yoptical splitter was collimated by using a lens (focal length of 11 $\mathrm{mm}$, Thorlabs, UK) and made normally incident at the sensor surface in order to resonantly excite PSPs on its corrugated gold surface. A flow-cell with a chamber depth of $100 \mu \mathrm{m}$, length $10 \mathrm{~mm}$, and width $5 \mathrm{~mm}$ was clamped against the gold sensor surface in order to contain aqueous samples. The flow cell consisted of a thin PDMS gasket and a transparent glass with drilled input and output ports. The analyzed liquid samples were flowed through the flowchamber by using a peristaltic pump (Ismatec, Switzerland) with a flow rate of $50 \mu \mathrm{L} / \mathrm{min}$. The light reflected from the sensor surface was coupled back to the Y-optical fiber splitter that was connected to a spectrometer (HR4000, OceanOptics Inc., USA). The measured spectrum of the light beam reflected from the grating surface was normalized with that acquired from the reference flat area on the sensor chip. The normalized reflectivity spectra $R(\lambda)$ were processed by a dedicated LabView software ${ }^{56}$ and the spectral position of the resonant coupling to PSPs $\lambda$ SPR was tracked as a function of 
time $t$ (Figure 2). The acquisition time of the SPR reflectivity spec- 62 tra was set at $7 \mathrm{~ms}$ and in each measurement spot, 100 spectra were 63 accumulated.

The changes in the spectral position of GC-SPR reflectivity dip 65 $\delta \lambda$ SPR were determined by fitting with an analytical function. The 66 resonant wavelength changes $\delta \lambda$ SPR were calibrated by the measur- 67 ing of sensor response to the flow of 1,2 and $4 \mathrm{wt} \%$ water solutions 68 of sucrose with known refractive indices $\left(\delta n_{s}=1.4 \times 10^{-3} ; 2.8 \times 10^{-} 69\right.$ $3 ; 5.6 \times 10^{-3} \mathrm{RIU}$, respectively) in order to convert the sensor signal 70 to refractive index units RIU. The refractive index sensitivity was 71 determined as $S=\delta \lambda_{\mathrm{SPR}} / \delta n_{\mathrm{s}}$. From the SPR signal baseline, the 72 standard deviation $\sigma[\lambda \operatorname{sPR}(t)]$ of its noise was obtained and the re- 73 fractive index resolution was obtained as $\sigma / S$.

Immobilization of aptamers. The HD1 or control aptamers 76 with amine terminal group were covalently coupled to the carbox- 77 ylic groups carried by the poly(HPMA-co-CBMAA) brushes. The 78 coupling was monitored in situ by the use of GC-SPR and firstly, 79 the baseline in $\lambda \operatorname{spR}(t)$ was established upon a flow of PBS (pH 7.4).

Then, the surface was incubated with $10 \mathrm{mM} \mathrm{SA}$ buffer $(\mathrm{pH}$ 5.0) 80 for $5 \mathrm{~min}$ and freshly prepared solution of EDC $(0.4 \mathrm{M})$ and NHS $(0.1 \mathrm{M})$ in $50 \mathrm{mM}$ MES buffer $(\mathrm{pH} 6.0)$ was reacted with the 81 brushes for $10 \mathrm{~min}$ in order to activate the carboxylic groups. Sub- 82 sequently, the surface was rinsed with $10 \mathrm{mM} \mathrm{SA}(\mathrm{pH} \mathrm{5.0)}$ and 83 HEPES ( $\mathrm{pH} 7.5$ ) for $1 \mathrm{~min}$ each. The activated surface was exposed 84 to $1 \mu \mathrm{M}$ solution of the aptamer for $30 \mathrm{~min}$ and rinsed with HEPES 85 (pH 7.5) for 5 min. Finally, the functionalized sensor surface was 86 washed with PBS ( $\mathrm{pH} 7.4$ ) for 90 min to let the unreacted active 87 ester groups hydrolyze.

Observation of the fouling. The fouling from undiluted HBP 89 was monitored by using GC-SPR instrument. Four types of GC- 90 SPR sensor chips with different surface chemistry were examined: 91 unmodified gold, gold surface with a mixed SAM (form up over- 92 night incubation in ethanolic solution with dissolved carboxyl 93 PEG-thiol and hydroxyl PEG-thiol at ratio 1:10, respectively, and 94 $1 \mathrm{mM}$ total concentration), gold surface with pristine poly(HPMA- 95 co-CBMAA) brushes and gold surface with poly(HPMA-co- 96 CBMAA) brushes that were functionalized with HD1 aptamer. For 97 each surface chemistry, a baseline in SPR signal $\lambda_{\mathrm{SPR}}(\mathrm{t})$ in PBS $(\mathrm{pH} 98$ 7.4) was established for $5 \mathrm{~min}$. Then, undiluted HBP that was 99 spiked with $1 \mu \mathrm{L}$ of argatroban monohydrate (stock $10 \mathrm{mg} / \mathrm{ml}$ ) in 00 final volume of $500 \mu \mathrm{L}$ was flowed over each surface for 15 minl 01 followed by the rinsing with PBS for $5 \mathrm{~min}$. The amount of unspe1 02 cifically bound species from HBP on the sensor surface was deter 103 mined in $\mathrm{ng} / \mathrm{mm}^{2}$ from the difference in the sensor signal beford 04 $\lambda_{\text {SPR }}$ and after the contact with the HBP by using the formula $\Gamma \geq 05$ $1.5 \cdot \delta \lambda_{\mathrm{SPR}}$ for polymer brushes and $0.85 \cdot \delta \lambda_{\mathrm{SPR}}$ for bare gold (SPR 06 wavelength shift $\delta \lambda_{\text {SPR }}$ is in $\mathrm{nm}$ ) based on numerical simulationd 07 discussed in the supporting information.

Detection of thrombin. The sensor chip with poly(HPMA-co 109 CBMAA) brushes functionalized with an aptamer ligand (specific 10 HD1 or scrambled HD1 sequence) were used to detect thrombin in 11 analyzed liquid samples (Figure 1b). There were prepared twd 12 types of samples that were spiked with a known concentration of 13 thrombin. The PBS sample was spiked with thrombin at a concen1 14 tration of $c=1,2.5,5,7.5,10,12.5,15,17.5,20,22.5$, and $25 \mathrm{nMl} 15$ $1 \mu \mathrm{L}$ of argatroban stock solution $(10 \mathrm{mg} / \mathrm{ml}, \mathrm{c}=19 \mathrm{mM})$ was added 16 to the analyzed HBP sample, gently mixed and incubated for $5 \min 117$ Afterwards, the HBP sample was spiked with thrombin (stock $50 \mathrm{~d} 18$ $\mathrm{nM}$ ) to obtain $c=5,10,15$ and $20 \mathrm{nM}$ in the final sample volume of 19 $500 \mu \mathrm{L}$ and gently mixed prior to the analysis by using GC-SPR 120 The measurement baseline in SPR sensor signal $\lambda \operatorname{SPR}(t)$ was estab121 lished upon a 5 min flow of PBS. Then, the sensor surface was exposed to the analyzed sample for $15 \mathrm{~min}$ and subsequently rinsed with PBS for $5 \mathrm{~min}$. In order to regenerate the surface and release the affinity bound thrombin, aqueous solution with $2 \mathrm{M} \mathrm{NaCl}$ was flowed for $2 \mathrm{~min}$. The sensor response $\triangle \mathrm{SPR}$ was determined in $\mu R I U$ from the difference in the SPR signal divided by refractive index sensitivity $\delta \lambda_{\mathrm{SPR}}(t) / S$ before and after the target analyte binding. Similarly, two types of control experiments were performed. All measurements carried in HBP were repeated three times in order to determine the standard deviation of the sensor response $\triangle \mathrm{SPR}$. The measured dependence of sensor response $\triangle \mathrm{SPR}$ on the thrombin concentration $c$ was fitted with Langmuir isotherm analytical function $\Delta \mathrm{SPR}=\Delta \mathrm{SPR}_{\max } \times c / K_{\mathrm{d}} /\left(1+c / K_{\mathrm{d}}\right)$ and where $\Delta \mathrm{SPR}_{\max }$ states for the response in saturation, $K_{\mathrm{d}}$ is the equilibrium dissociation affinity constant. The limit of detection (LOD) was defined as a concentration where 3 times the standard deviation of the baseline signal $3 \times \sigma[\lambda \operatorname{sPR}(t)]$ intersects the Langmuir fit of the calibration curve.

\section{RESULTS AND DISCUSSION}

Characterization of the compact sensor and its chips. The crossed relief grating structure was fabricated by using the UVnanoimprint lithography followed by the coating with $100 \mathrm{~nm}$ thick gold layer in order to resonantly couple incident light beam to PSPs. The polymer brushes were subsequently synthesized on top of the gold film in order to provide biointerface resistant to fouling from HBP. The surface of the diffraction grating with and without polymer brushes was analyzed by AFM as shown in Figure 1c. The period of the gold-coated grating with sinusoidal profile was determined as $\Lambda=434 \pm 16 \mathrm{~nm}$ and the corrugation depth $h=29.5 \pm 3.5 \mathrm{~nm}$. After the synthesis of the polymer brushes with a thickness of $d=35 \pm 7 \mathrm{~nm}$, the grating corrugation depth decreased to $d=13.2 \pm 1.6 \mathrm{~nm}$ and reduced roughness of the topography was observed (Figure S1). These phenomena can be explained by the fact that after their drying, the flexible polymer chains rearrange and tends to fill the concave areas of the grating.

The plasmonic grating structure on the top of the sensor chip was attached to a flow cell and loaded to the compact optical reader (see Figure 2a). The resonant excitation of PSPs on the bare gold grating is manifested as a narrow dip in the wavelength reflectivity spectrum $R(\lambda)$. It occurs at a wavelength $\lambda_{\text {SPR, }}$, where the normally incident wave and PSPs are phase-matched due to the diffraction on the grating periodic corrugation. The momentum of the PSP waves is equal to the grating momentum $\operatorname{Re}\{k \operatorname{PsP}(\lambda)\}=2 \pi / \Lambda$ for the herein used first diffraction order coupling, which is fulfilled at a wavelength $\lambda_{\mathrm{SPR}}=635.8 \mathrm{~nm}$ (see the spectral position of the reflectivity dip in Figure 2b). After the synthesis of poly(HPMA-co-CBMAA) brushes on the top of the gold grating, the resonant wavelength shifts to $\lambda_{\mathrm{SPR}}=655.1 \mathrm{~nm}$ due to an increase of the surface mass density $\Gamma$.

Importantly, the presence of polymer brushes (with a thickness of $d=35 \mathrm{~nm}$ ) affects the sensitivity of the SPR sensor $S$ to the refractive index changes occurring on its surface. The reason is that the polymer occupies part of the volume that is probed by the evanescent field of PSPs with a decay length of about $L_{\mathrm{p}} \sim 100 \mathrm{~nm}$. By measuring the detuning of $\lambda_{\mathrm{SPR}}$ due to the refractive index change $n_{\mathrm{s}}$ of an aqueous solution spiked with sucrose (Figure S2), the sensitivity of $S=459 \pm 20 \mathrm{~nm} / \mathrm{RIU}$ was determined for the GC-SPR chip without the polymer brushes. This value agrees with previous GC-SPR measurements at a similar wavelength..$^{56}$ On the same chips that carried the polymer brushes biointerface architecture the sensitivity decreased about twice to $S=238.3 \pm 5.4 \mathrm{~nm} / \mathrm{RIU}$. The 
1 baseline of the SPR sensor signal exhibited the noise with the stand2 ard deviation of $\sigma=3 \times 10^{-3} \mathrm{~nm}$, which translates to the instrument 3 refractive index resolution of $6 \times 10^{-6}$ RIU without the brushes and $41.1 \times 10^{-5} \pm$ RIU with the brushes. It is worth of noting that more
5 than an order of magnitude better resolution can be achieved with more advanced engineering of GC-SPR sensor instrument, how7 ever, then more sophisticated stabilization (e.g. temperature) has to 8 be implemented. ${ }^{29}$ a) Compact reader

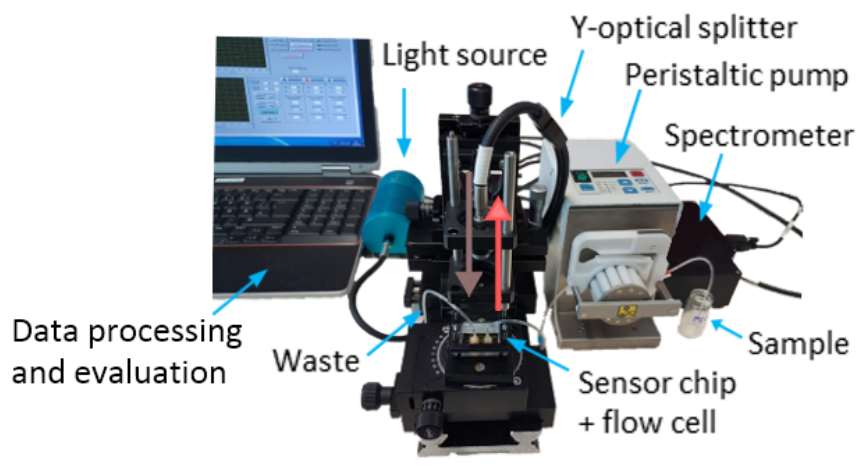

b) Reflectivity spectra $R(\lambda)$

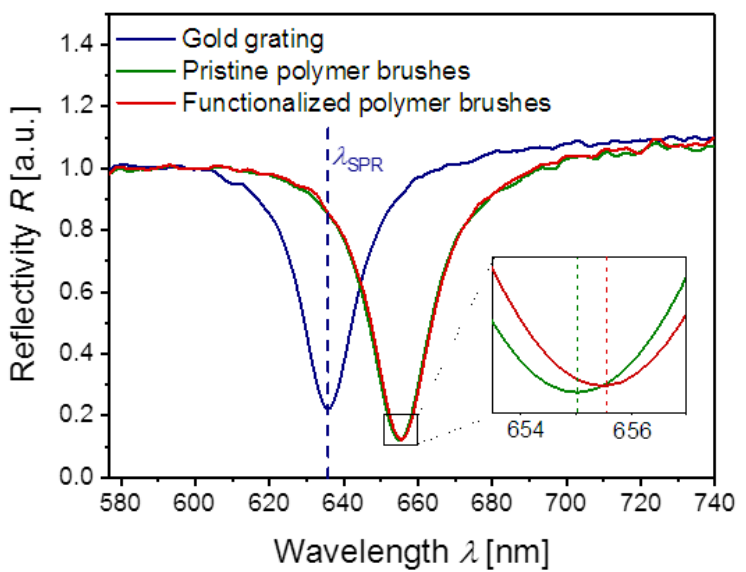

c) SPR kinetics $\lambda_{\mathrm{SPR}}(t)$

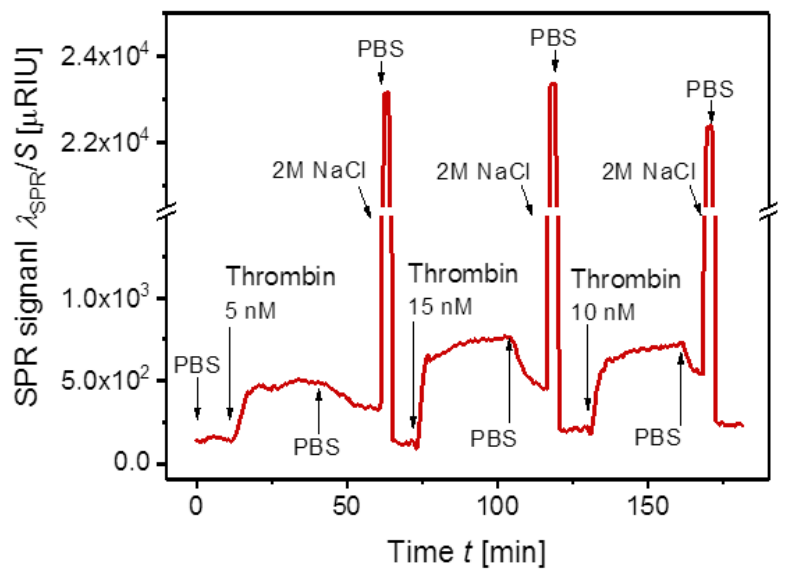

Figure 2. a) A photograph of the compact GC-SPR instrument. b) The SPR spectra obtained from the gold grating, grating modified with pristine polymer brushes and after the immobilization of the aptamer and c) The sensorgram showing detection of thrombin in PBS by using a plasmonic grating chip modified with poly(HPMA-co-CBMAA) brush and HD1 aptamer.

Immobilization of the aptamers. In order to allow for the spe- 31 cific capture of target analyte at the sensor surface, the aptamer re- 32 ceptors were tethered to polymer brushes. The immobilization of 33 thrombin-specific HD1 aptamer or scrambled control aptamer was 34 performed in-situ and monitored by GC-SPR. The reflectivity spec- 35 tra $R(\lambda)$ (Figure $2 \mathrm{~b}$ ) and respective SPR sensor signal kinetics 36 $\lambda_{\operatorname{SPR}}(t)$ (Figure S3a) show that after the immobilization of the ap- 37 tamer, the resonance shifts to longer wavelengths. After $30 \mathrm{~min}, 38$ non-covalently bound aptamers were washed from the surface with 39 a PBS buffer. A shift in the position of the SPR resonance $\Delta \lambda \lambda_{\text {SPR }}$ of 40 about $0.5 \mathrm{~nm}$ was observed after the immobilization of the HD1 41 aptamer on the sensor surface which corresponds to the $2.3 \pm 0.942$ mRIU for HD1 thrombin specific aptamer and $2.4 \pm 0.1 \mathrm{mRIU}$ for 43 the scrambled sequence of HD1 aptamer (Figure S3b).

Investigation of the fouling of biointerface architectures. 45 The fouling from undiluted HBP was observed from SPR signal 46 measured upon the contact of the sensor surface with a sample and it was quantified in terms of the surface mass density change $\Gamma$ determined after the rinsing of the surface with PBS. Besides the polymer brushes-coated gold grating surfaces, also a sensor chips carrying a layer of bare gold and gold modified with PEG-thiol SAM were examined for comparison. Figure 3a shows the SPR sensor signal measured upon the $15 \mathrm{~min}$ flow of HBP over each type of surface followed by the rinsing. These data show that the injection of HPB rapidly shifts the SPR wavelength due to the bulk refractive index change $\Delta n_{\mathrm{s}}$. Afterwards, there is observed a slower gradual increase in the SPR signal on the bare and PEG-thiol modified gold surface due to the adsorption of HPB constituents. Importantly, much smaller gradual changes in SPR signal are measured on the polymer brushes, which indicates weaker unspecific interaction with HBP. After the rinsing with PBS, the SPR signal rapidly decreases owing to a change in the bulk refractive index $\Delta n_{\mathrm{s}}$ and it 
reaches an equilibrium after $5 \mathrm{~min}$. The surface mass density of the 28 irreversibly deposited constituents $\Gamma$ was determined from the 29 change in the SPR signal baseline before and after the exposure to 30 HBP as summarized in Figure $3 \mathrm{~b}$. In agreement with the previous 31 studies, ${ }^{46}$ there was observed no measurable change in $\Gamma$ for pris- 32 tine brushes demonstrating that the anti-fouling properties are not 33 affected by the grating topology. These characteristics are superior 34 to the bare gold $\left(\Gamma=0.66 \mathrm{ng} / \mathrm{mm}^{2}\right)$ and conventional gold modified 35 with PEG-thiol SAM $\left(\Gamma=0.59 \mathrm{ng} / \mathrm{mm}^{2}\right)$.

a)

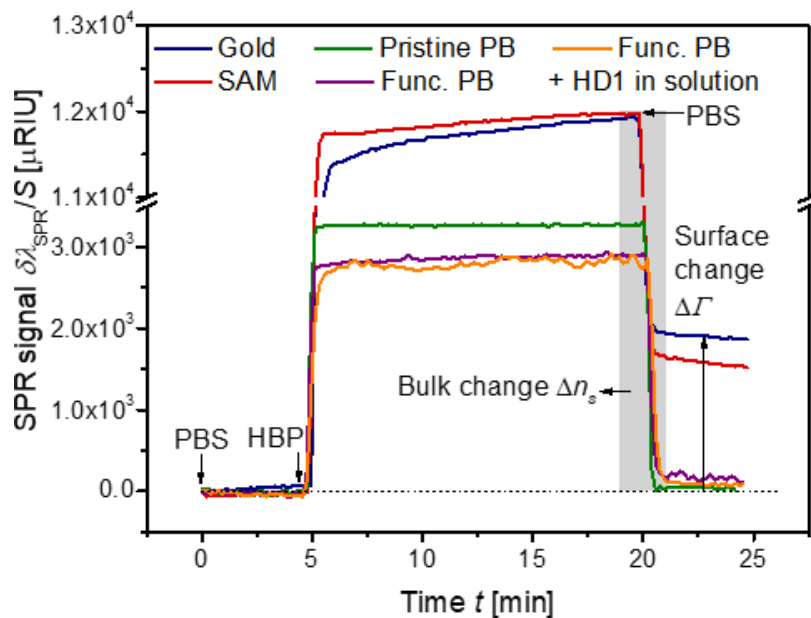

b)

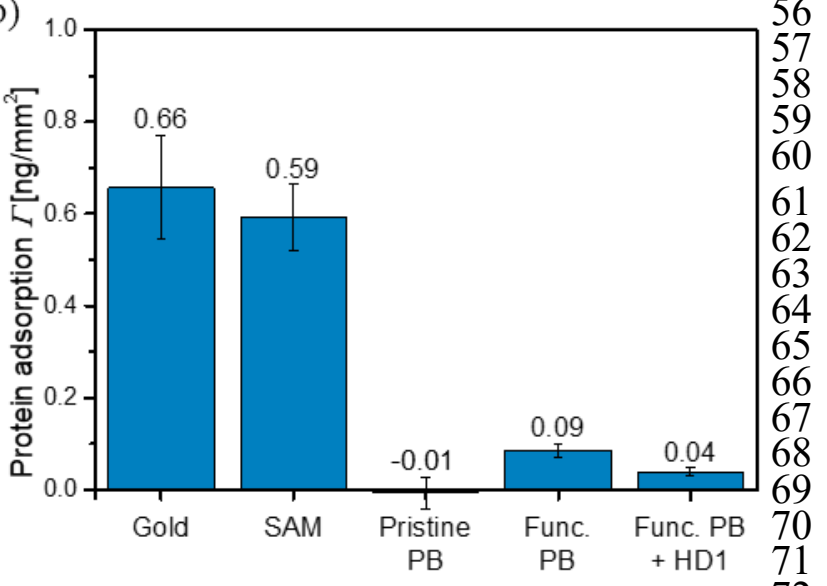

10

Figure 3. a) The sensorgram showing fouling of the examined sur- 74 SAM - gold modified with a thiol-based SAM, pristine PB - gold 76 modified with polymer brushes, func. PB - gold modified with pol- 77 averaged protein adsorption $\Gamma$ reflecting fouling of each surface to 79 $100 \%$ plasma with $38 \mu \mathrm{M}$ argatroban.

However, in contrast to previous study ${ }^{45}$ (in which a protein lig- 81 and was immobilized to similar brushes) we observed a significant 82 increase in the surface mass density $\left(\Gamma=0.09 \mathrm{ng} / \mathrm{mm}^{2}\right)$ for the 83 brushes that were functionalized with the HD1 DNA aptamer. This 84 observation indicates that the presence of negatively charged DNA 85 strands can impair more severely the anti-fouling properties, but it 86 also can be attributed to the capture of endogenous thrombin that is 87 natively present in the tested pooled $\mathrm{HBP}$ at $\mathrm{pM}$ concentrations. ${ }^{57,58} 88$ In order to elucidate between these two possible contributions, we also measured the fouling for the HD1 aptamer-functionalized brushes from HBP that was spiked with the same HD1 aptamer. Then, we observed about two-times lower surface mass density increase $\left(\Gamma=0.04 \mathrm{ng} / \mathrm{mm}^{2}\right)$, which can be ascribed to the blocking of the thrombin present in the sample and thus preventing its specific capture by the sensor HD1 aptamer tethered to the surface. Therefore, about $50 \%$ of the observed SPR response can be ascribed to the fouling and it should be highlighted that it corresponds to about $7 \%$ of the value observed on (even non-functionalized) PEG-thiol SAM that is widely accepted as standard low-fouling surface architecture. faces to $100 \%$ plasma with $38 \mu \mathrm{M}$ argatroban: gold - bare gold, 75 ymer brushes that were functionalized with HD1 aptamer. b) The 78
Detection of thrombin. The developed GC-SPR sensor chip with poly(HPMA-co-CBMAA) brushes was used for the detection of thrombin in model PBS and undiluted HBP samples. The polymer brushes were functionalized either with thrombin-specific aptamer HD1 or by an aptamer with a scrambled sequence of nucleotides in order to serve as a negative control. There were prepared a series of HBP samples spiked with $38 \mu \mathrm{M}$ argatroban and thrombin in the concentration range from $c=1$ to $25 \mathrm{nM}$. The impact of argatroban on a thrombin detection assay was investigated and it was confirmed that the argatroban does not interfere with the aptamer binding site (Figure S4). As can be seen in Figure 2c and Figure 4a, prepared samples were subsequently analyzed with the SPR sensor signal $\lambda_{\mathrm{SPR}} / S$ aquired in time. Each sample was flowed through the sensor for 15 min followed by rinsing with PBS and regeneration of the surface by introducing an aqueous solution of $\mathrm{NaCl}(2 \mathrm{M})$, which weakens the thrombin-HD1 affinity binding. Importantly, the regeneration has been complete and lead to washing off all of the specifically captured thrombin as well as unspecifically adsorbed abundant HBP constituents. The control experiment was performed analogously in sensor chips with the polymer brushes functionalized with a scrambled sequence of the HD1 aptamer that does not form a G-quadruplex structure which is indispensable for the binding of thrombin.

As there is indicated in Figure 4a, the sensor response $\triangle \mathrm{SPR}$ due to the binding of thrombin was determined from the SPR signal changes before the injection of a sample and after the rising with PBS prior to the regeneration step. The calibration curves were established for detection of thrombin in PBS (blue symbols in Figure 4b) and HBP (red symbols in Figure 4b). These data were fitted by using the Langmuir isotherm and the obtained dissociation equilibrium constant were of $K_{\mathrm{d}}=3.7$ and $22 \mathrm{nM}$ for the PBS and HBP samples, respectively. Such variation can be attributed to potential interaction of immobilized HD1 aptamer with other constituents that are present in HBP and not in PBS. These can include the background thrombin level in used pooled plasma (which would lead to false concentration of thrombin) and to the presence of ions and macromolecules that amend the balance of aptamer intermolecular interactions. It is worth of noting that the obtained $K_{\mathrm{d}}$ values fall in the range that was reported in the literature and measured by using the aptamers utilized for heterogeneous assays ${ }^{59,60}$ as well as homogeneous assays. ${ }^{61,62}$ By taking into account the noise of the sensor signal, the limit of detection was determined as $\mathrm{LOD}=0.15$ and $1.1 \mathrm{nM}$ for the PBS and HBP, respectively. In addition, squares in Figure $4 \mathrm{~b}$ represent two control experiments obtained with the scrambled aptamer sequence in PBS (blue color) and human plasma (red color). An additional control experiment was performed by using HBP samples containing $38 \mu \mathrm{M}$ argatroban that were spiked with $1 \mu \mathrm{M}$ HD1 or scrambled control aptamer (Figure S5). The obtained data are in the good agreement with the values that were obtained in the direct assay in HBP. 
a)

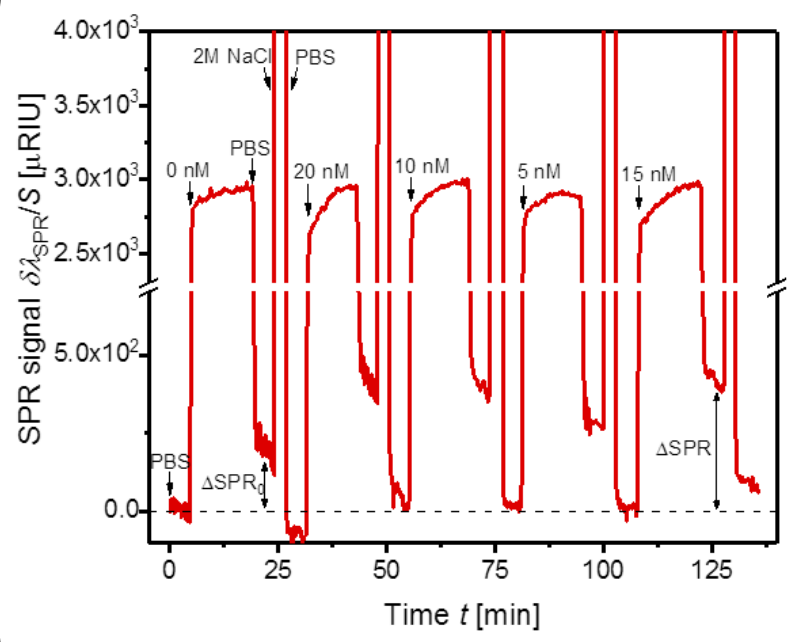

b)

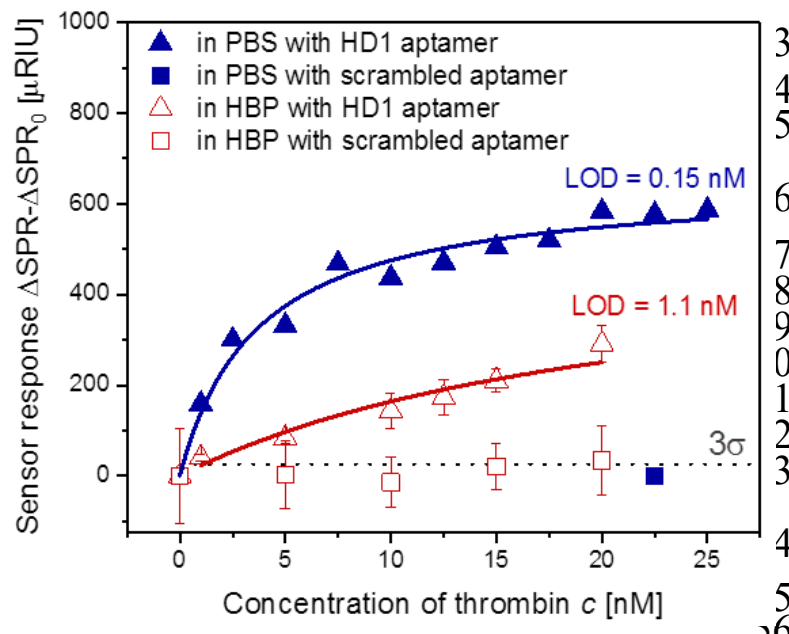

2 Figure 4. a) The sensorgram showing detection of thrombin in 58 $3100 \%$ plasma. b) The normalized SPR response vs. concentration 59 4 of thrombin measured in PBS (blue) $(\mathrm{n}=1)$ and $100 \%$ plasma (red) 60 $5 \quad(\mathrm{n}=3)$ by using the brushes functionalized with HD1 aptamer (tri- 61 6 angles) or with control aptamer (squares).

\section{CONCLUSIONS}

An optical biosensor platform for rapid direct monitoring of 68 thrombin in HBP was established based on grating-coupled SPR 69

\section{ASSOCIATED CONTENT}

Supporting Information Available: The following files are available free of charge. Kotlarek_ACS Sensors_supporting information.pdf. It contains information on the topography changes of grating corrugation after the coating with antifouling polymer brushes and calibration of the sensor. In addition, GC-SPR sensorgrams for the functionalization of sensor chips are provided together with an impact of argatroban to the assay performance and control thrombin assay. AUTHOR INFORMATION

\section{Corresponding Author}

Jakub Dostalek, PhD, E-mail: jakub.dostalek@ait.ac.at, Phone: +43 (0) 50550 4470, Fax: +43 (0) 505504450.

\section{ACKNOWLEDGMENT}

This project has received funding from the European Union's Horizon 2020 research and innovation programme under grant agreement No 642787, Marie Sklodowska-Curie Innovative Training Network BIOGEL. CR-E and M.V. acknowledge the support of the Deutsche Forschungsgemeinschaft (DFG) in the framework of the priority programme 2014 "Towards an Implantable Lung", project numbers: 346972946 and 347367912 ".

\section{REFERENCES}

(1) Rasche, H. Haemostasis and Thrombosis: An Overview. Eur. Hear. Journal, Suppl. 2001, 3 (Q), 3-7.

(2) Sira, J.; Eyre, L. Physiology of Haemostasis. Anaesth. Intensive Care Med. 2016, 17 (2), 79-82.

(3) Miyares, M. A.; Davis, K. Newer Oral Anticoagulants: A Review of Laboratory Monitoring Options and Reversal Agents in the Hemorrhagic Patient. Am. J. Heal. Pharm. 2012, 69 (17), 1473-1484.

(4) Adams, G. L.; Manson, R. J.; Turner, I.; Sindram, D.; Lawson, J. H. The Balance of Thrombosis and Hemorrhage in Surgery. Hematol. Oncol. Clin. North Am. 2007, 21 (1), 13-24.

(5) Falck-Ytter, Y.; Francis, C. W.; Johanson, N. A.; Curley, C.; Dahl, O. E.; Schulman, S.; Ortel, T. L.; Pauker, S. G.; Colwell, C. W. Prevention of VTE in Orthopedic Surgery Patients: Antithrombotic Therapy and Prevention of Thrombosis. Chest 2012, 141 (2), e278S-e325S.

(6) Edmunds, L. H.; Colman, R. W. Thrombin During Cardiopulmonary Bypass. Ann. Thorac. Surg. 2006, 82 (6), 2315-2322.

(7) Mazzeffi, M.; Greenwood, J.; Tanaka, K.; Menaker, J.; Rector, R.; Herr, D.; Kon, Z.; Lee, J.; Grif, B.; Rajagopal, K.; et al. Bleeding, Transfusion, and Mortality on Extracorporeal Life Support: ECLS Working Group on Thrombosis and Hemostasis. Ann. Thorac. Surg. 2016, 101(2), $682-689$.

(8) Cortelazzo, S.; Finazzi, G.; Viero, P.; Galli, M.; Remuzzi, A.; Parenzan, L.; Barbui, T. Thrombotic and Hemorrhagic Complications in Patients with Mechanical Heart Valve Prosthesis Attending an Anticoagulation Clinic. Thromb. Haemost. 1993, 70 (04), 316-320.

(9) Despotis, G. J.; Joist, J. H.; Goodnough, L. T. Monitoring of Hemostasis in Cardiac Surgical Patients: Impact of Point- of-Care Testing on Blood Loss and Transfusion Outcomes. Clin. Chem. 1997, 43 (9), 16841696.

(10) Heneghan, C.; Alonso-Coello, P.; Garcia-Alamino, J. M.; Perera, R.; Meats, E.; Glasziou, P. Self-Monitoring of Oral Anticoagulation: A 
1 Systematic Review and Meta-Analysis. Lancet 2006, 367 (9508), 404-411. 72 (11) Bashaw, M. Coagulopathy In and Outside the Intensive Care Unit. 73 Crit. Care Nurs. Clin. NA 2017, 29 (3), 353-362.

(12) Zheng, R.; Cameron, B. D. Surface Plasmon Resonance: Recent 75 Progress toward the Development of Portable Real-Time Blood 76 Diagnostics. Expert Rev. Mol. Diagn. 2012, 12 (1), 5-7.

(13) Tokel, O.; Inci, F.; Demirci, U. Advances in Plasmonic 78 Technologies for Point of Care Applications. Chem. Rev. 2014, 114 (11), 79 $5728-5752$.

(14) Feltis, B. N.; Sexton, B. A.; Glenn, F. L.; Best, M. J.; Wilkins, M.; 81 Davis, T. J. A Hand-Held Surface Plasmon Resonance Biosensor for the 82 Detection of Ricin and Other Biological Agents. Biosens. Bioelectron. 83 2008, 23 (7), 1131-1136.

(15) Wang, S.; Xie, J.; Jiang, M.; Chang, K.; Chen, R.; Ma, L.; Zhu, J.; 85 Guo, Q.; Sun, H.; Hu, J. The Development of a Portable SPR Bioanalyzer 86 for Sensitive Detection of Escherichia Coli O157:H7. Sensors (Switzerland) 87 2016, 16 (11), 1-9.

(16) Mauriz, E.; Calle, A.; Montoya, A.; Lechuga, L. M. Determination 89 of Environmental Organic Pollutants with a Portable Optical 90 Immunosensor. Talanta 2005, 69 (2 SPEC. ISS.), 359-364.

(17) Soelberg, S. D.; Furlong, C. E. Biosensors and Bioassays for 92 Ecological Risk Monitoring and Assessment. In Environmental Toxicology; 93 Springer: New York, 2013; pp 121-142.

(18) Prabowo, B. A.; Wang, R. Y. L.; Secario, M. K.; Ou, P. T.; Alom, 95 A.; Liu, J. J.; Liu, K. C. Rapid Detection and Quantification of Enterovirus 96 71 by a Portable Surface Plasmon Resonance Biosensor. Biosens. 9 Bioelectron. 2017, 92, 186-191.

(19) Trzaskowski, M.; Napiórkowska, A.; Augustynowicz-Kopeć, E.: 99 Ciach, T. Detection of Tuberculosis in Patients with the Use of Portabld 00 SPR Device. Sensors Actuators, B Chem. 2018, 260, 786-792.

(20) Naimushin, A. N.; Soelberg, S. D.; Bartholomew, D. U.; Elkind, Jl 02 L.; Furlong, C. E. A Portable Surface Plasmon Resonance (SPR) Sensol 03 System with Temperature Regulation. Sensors Actuators, B Chem. 2003, $9 d 04$ (1-2), 253-260.

105

(21) Stevens, R. C.; Soelberg, S. D.; Near, S.; Furlong, C. E. Detection 06 of Cortisol in Saliva with a Flow-Filtered, Portable Surface Plasmon 07 Resonance Biosensor System. Anal. Chem. 2008, 80 (17), 6747-6751. 108

(22) Dostálek, J.; Homola, J.; Miler, M. Rich Information Formal 09 Surface Plasmon Resonance Biosensor Based on Array of Diffraction 10 Gratings. Sensors Actuators, B Chem. 2004, 107 (1 SPEC. ISS.), 154-1611 11

(23) Wang, Y.; Dostalek, J.; Knoll, W. Magnetic Nanoparticle1 12 Enhanced Biosensor Based on Grating-Coupled Surface Plasmon 13 Resonance. 2011, 6202-6207.

(24) Arachchillage, D. R.; Passariello, M.; Laffan, M.; Aw, T. C.115 Owen, L.; Banya, W.; Trimlett, R.; Morgan, C.; Patel, B. V.; Pepper, J.; el 16 al. Intracranial Hemorrhage and Early Mortality in Patients Receiving 17 Extracorporeal Membrane Oxygenation for Severe Respiratory Failurel 18 Semin. Thromb. Hemost. 2018, 44 (03), 276-286.

(25) Kooy, N.; Mohamed, K.; Pin, L. T.; Guan, O. S. A Review of Roll120 to-Roll Nanoimprint Lithography | Nanoscale Research Letters | Full Text1 21 Nanoscale Res. Lett. 2014, 9 (1), 1.

(26) Lu, C.; Lipson, R. H. Interference Lithography: A Powerful Tool 23 for Fabricating Periodic Structures. Laser Photonics Rev. 2010, 4 (4), 568124 580.

(27) Vala, M.; Chadt, K.; Piliarik, M.; Homola, J. High-Performancd 26 Compact SPR Sensor for Multi-Analyte Sensing. Sensors Actuators, 127 Chem. 2010, 148 (2), 544-549.

(28) Fernández, F.; Hegnerová, K.; Piliarik, M.; Sanchez-Baeza, F.129 Homola, J.; Marco, M. P. A Label-Free and Portable Multichannel Surfacd 30 Plasmon Resonance Immunosensor for on Site Analysis of Antibiotics in 3 Milk Samples. Biosens. Bioelectron. 2010, 26 (4), 1231-1238.

(29) Piliarik, M.; Homola, J. Surface Plasmon Resonance (SPR) 33 Sensors: Approaching Their Limits? Opt. Express 2009, 17 (19), 16505. 134

(30) Zhang, J.; Khan, I.; Zhang, Q.; Liu, X.; Dostalek, J.; Liedberg, B.135 Wang, Y. Lipopolysaccharides Detection on a Grating-Coupled Surfacd 36 Plasmon Resonance Smartphone Biosensor. Biosens. Bioelectron. 2018, 943 (April 2017), 312-317.

(31) Centi, S.; Tombelli, S.; Minunni, M.; Mascini, M. Aptamer-Based 39 Detection of Plasma Proteins by an Electrochemical Assay Coupled td 40 Magnetic Beads. Anal. Chem. 2007, 79 (4), 1466-1473.

(32) Bini, A.; Minunni, M.; Tombelli, S.; Centi, S.; Mascini, Ml 42
Analytical Performances of Aptamer-Based Sensing for Thrombin Detection. Anal. Chem. 2007, 79 (7), 3016-3019.

(33) Pavlov, V.; Xiao, Y.; Shlyahovsky, B.; Willner, I. AptamerFunctionalized Au Nanoparticles for the Amplified Optical Detection of Thrombin. J. Am. Chem. Soc. 2004, 126 (38), 11768-11769.

(34) Baek, S. H.; Wark, A. W.; Lee, H. J. Dual Nanoparticle Amplified Surface Plasmon Resonance Detection of Thrombin at Subattomolar Concentrations. Anal. Chem. 2014, 86 (19), 9824-9829.

(35) He, P.; Liu, L.; Qiao, W.; Zhang, S. Ultrasensitive Detection of Thrombin Using Surface Plasmon Resonance and Quartz Crystal Microbalance Sensors by Aptamer-Based Rolling Circle Amplification and Nanoparticle Signal Enhancement. Chem. Commun. 2014, 50 (12), 14811484.

(36) Li, S.; Zhang, D.; Zhang, Q.; Lu, Y.; Li, N.; Chen, Q.; Liu, Q. Electrophoresis-Enhanced Localized Surface Plasmon Resonance Sensing Based on Nanocup Array for Thrombin Detection. Sensors Actuators, B Chem. 2016, 232, 219-225.

(37) Lao, J.; Han, L.; Wu, Z.; Zhang, X.; Huang, Y.; Tang, Y.; Guo, T. Gold Nanoparticle-Functionalized Surface Plasmon Resonance Optical Fiber Biosensor: In Situ Detection of Thrombin with 1 NM Detection Limit. J. Light. Technol. 2019, 37 (11), 2748-2755.

(38) Andrade, J. D. Surface and Interfacial Aspects of Biomedical Polymers: Volume 1 Surface Chemistry and Physics; Springer Science \& Business Media, 2012.

(39) Vroman, L.; Adams, A. L.; Fischer, G.; Munoz, P. Interaction of High Molecular Weight Kininogen, Factor XII, and Fibrinogen in Plasma at Interfaces. Blood 1980, 55 (1), 156-159.

(40) Ostuni, E.; Chapman, R. G.; Holmlin, R. E.; Takayama, S.; Whitesides, G. M. A Survey of Structure-Property Relationships of Surfaces That Resist the Adsorption of Protein. Langmuir 2001, 17 (18), $5605-5620$

(41) Emmenegger, C. R.; Brynda, E.; Riedel, T.; Sedlakova, Z.; Houska, M.; Alles, A. B. Interaction of Blood Plasma with Antifouling Surfaces. Langmuir 2009, 25 (11), 6328-6333.

(42) Polonschii, C.; David, S.; Tombelli, S.; Mascini, M.; Gheorghiu, M. A Novel Low-Cost and Easy to Develop Functionalization Platform. Case Study: Aptamer-Based Detection of Thrombin by Surface Plasmon Resonance. Talanta 2010, 80 (5), 2157-2164.

(43) Bai, Y.; Feng, F.; Zhao, L.; Wang, C.; Wang, H.; Tian, M.; Qin, J.; Duan, Y.; He, X. Aptamer/Thrombin/Aptamer-AuNPs Sandwich Enhanced Surface Plasmon Resonance Sensor for the Detection of Subnanomolar Thrombin. Biosens. Bioelectron. 2013, 47, 265-270.

(44) Špringer, T.; Bocková, M.; Homola, J. Label-Free Biosensing in Complex Media: A Referencing Approach. Anal. Chem. 2013, 85 (12) $5637-5640$

(45) Riedel, T.; Surman, F.; Hageneder, S.; Pop-Georgievski, O.; Noehammer, C.; Hofner, M.; Brynda, E.; Rodriguez-Emmenegger, C.; Dostálek, J. Hepatitis B Plasmonic Biosensor for the Analysis of Clinical Serum Samples. Biosens. Bioelectron. 2016, 85, 272-279.

(46) Riedelová, Z.; Májek, P.; Pečánková, K.; Kučerová, J.; Surman, F.; De Los Santos Pereira, A.; Riedel, T. SPR Biosensor for Quantification of Fetuin-A as a Promising Multibiomarker. 2018, 67, 367-375.

(47) Vorobii, M.; Kostina, N. Y.; Rahimi, K.; Grama, S.; Söder, D. Pop-Georgievski, O.; Šturcová, A.; Horák, D.; Grottke, O.; Singh, S.; et al. Antifouling Microparticles to Scavenge Lipopolysaccharide from Human Blood Plasma. 2019, 20 (2), 959-968.

(48) Riedel, T.; Hageneder, S.; Surman, F.; Pop-Georgievski, O.; Noehammer, C.; Hofner, M.; Brynda, E.; Rodriguez-Emmenegger, C.; Dostálek, J. Plasmonic Hepatitis B Biosensor for the Analysis of Clinical Saliva. Anal. Chem. 2017, 89 (5), 2972-2977.

(49) Bock, L. C., Griffin, L. C., Latham, J. A., Vermaas, E. H., \& Toole, J. J. Selection of Single-Stranded DNA Molecules That Bind and Inhibit Human Thrombin. Nature 1992, 355 (6360), 564-566.

(50) Paborskys, L. R.; Mccurdy, S. N.; Griffin, L.; Toole, J. J.; Lawrence, L.; Leung, K. The Single-Stranded DNA Aptamer-Binding Site of Human Thrombin. 1993, 20808-20811.

(51) Di Nisio, M.; Middeldorp, S.; Büller, H. R. Direct Thrombin Inhibitors. N. Engl. J. Med. 2005, 353 (10), 1028-1040.

(52) Bauch, M.; Hageneder, S.; Dostalek, J. Plasmonic Amplification for Bioassays with Epi-Fluorescence Readout. Opt. Express 2014, 22 (26), 32026. 
(53) Jones, D. M.; Brown, A. A.; Huck, W. T. S.; Street, P.; Cb, C. 24 Surface-Initiated Polymerizations in Aqueous Media: Effect of Initiator 25 Density. 2002, No. 13, 1265-1269.

(54) Ulbrich, K.; Šubr, V.; Strohalm, J.; Plocová, D.; Jelínková, M.; 27 Ríhová, B. Polymeric Drugs Based on Conjugates of Synthetic and Natural 28 Macromolecules. J. Control. Release 2000, 64 (1-3), 63-79.

(55) Rodriguez-Emmenegger, C.; Schmidt, B. V. K. J.; Sedlakova, Z.; 30 Šubr, V.; Alles, A. B.; Brynda, E.; Barner-Kowollik, C. Low Temperature 31 Aqueous Living/Controlled (RAFT) Polymerization of Carboxybetaine 32

10 Methacrylamide up to High Molecular Weights. Macromol. Rapid 33 Commun. 2011, 32 (13), 958-965.

(56) Reiner, A. T.; Ferrer, N. G.; Venugopalan, P.; Lai, R. C.; Lim, S. 35 3 K.; Dostálek, J. Magnetic Nanoparticle-Enhanced Surface Plasmon 36 14 Resonance Biosensor for Extracellular Vesicle Analysis. Analyst 2017, 14237 (20), 3913-3921.

(57) Hemker, H. C.; Beguin, S. The Clotting Mechanism and How to 39 Determine Its Phenotype. Arch. Hell. Med. 2000, 17, 13-18.

(58) Müller, J.; Becher, T.; Braunstein, J.; Berdel, P.; Gravius, S.; 41 Rohrbach, F.; Oldenburg, J.; Mayer, G.; Pötzsch, B. Profiling of Active 42 Thrombin in Human Blood by Supramolecular Complexes. Angew. Chemie 43 - Int. Ed. 2011, 50 (27), 6075-6078.

(59) Müller, J.; Freitag, D.; Mayer, G.; Pötzsch, B. Anticoagulant 45 23 Characteristics of HD1-22, a Bivalent Aptamer That Specifically Inhibits
Thrombin and Prothrombinase. J. Thromb. Haemost. 2008, 6 (12), 2105 2112.

(60) Pasternak, A.; Hernandez, F. J.; Rasmussen, L. M.; Vester, B.; Wengel, J. Improved Thrombin Binding Aptamer by Incorporation of a Single Unlocked Nucleic Acid Monomer. Nucleic Acids Res. 2011, 39 (3), 1155-1164.

(61) Li, J. J.; Fang, X.; Tan, W. Molecular Aptamer Beacons for RealTime Protein Recognition. Biochem. Biophys. Res. Commun. 2002, 292 (1), $31-40$.

(62) Zavyalova, E.; Tagiltsev, G.; Reshetnikov, R.; Arutyunyan, A.; Kopylov, A. Cation Coordination Alters the Conformation of a ThrombinBinding G-Quadruplex DNA Aptamer That Affects Inhibition of Thrombin. Nucleic Acid Ther. 2016, 26 (5), 299-308.

(63) Hemker, H. C.; Beguin, S. Thrombin Generation in Plasma: Its Assessment via the Endogenous Thrombin Potential. Thromb. Haemost. 1995, 74 (1), 134-138.

(64) Mann, K. G.; Brummel, K.; Butenas, S. What Is All That Thrombin For? J. Thromb. Haemost. 2003, 1 (7), 1504-1514.

(65) Brummel, K. E.; Paradis, S. G.; Butenas, S.; Mann, K. G. Thrombin Functions during Tissue Factor-Induced Blood Coagulation. Blood 2002, 100 (1), 148-152. 
SYNOPSIS TOC
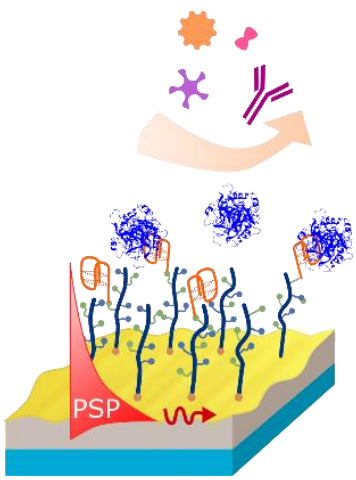

For TOC only 